\title{
Comparative analysis of prokaryotic and eukaryotic transcription factors using machine-learning techniques
}

\author{
Nilkanta Chowdhury \& Angshuman Bagchi* \\ ${ }^{1}$ Department of Biochemistry and Biophysics, University of Kalyani, Kalyani, Nadia 741235, India. Angshuman Bagchi - E-mail: \\ angshu@klyuniv.ac.in, angshuman_bagchi@yahoo.com; Telephone: +919051948843; Fax: +913325828282; *Corresponding author
}

Received June 24, 2018; Revised June 28, 2018; Accepted June 28, 2018; Published June 30, 2018

doi:10.6026/97320630014315

\begin{abstract}
:
The DNA-protein interactions play vital roles in the central dogma of molecular biology. Proper interactions between DNA and protein would lead to the onset of various biological phenomena like transcription, translation, and replication. However, the mechanisms of these well-known processes vary between prokaryotic and eukaryotic organisms. The exact molecular mechanisms of these processes are unknown. Therefore, it is of interest to report the comparative estimate of the different properties of the DNA binding proteins from prokaryotic and eukaryotic organisms. We analyzed the different sequence-based features such as the frequency of amino acids and amino acid groups in the proteins of prokaryotes and eukaryotes by statistical measures. The general pattern of differences between the various DNA binding proteins for the development of a prediction system to discriminate between these proteins between prokaryotes and eukaryotes is documented.
\end{abstract}

Keywords: Prokaryotic and Eukaryotic Organisms; DNA binding proteins; Transcription factors; Distribution of amino acid residues.

\section{Background:}

DNA protein interactions as in DNA transcription are at the heart of the central dogma of molecular biology. The transcription is the process of transfer of genetic information from DNA molecules. The process is regulated by a set of proteins. These proteins are referred to as the transcription factors (TFs) [1]. The mechanism of the process is a very complex one and is mainly mediated by a complex interplay between the TFs with DNA. However, the mechanism of DNA transcription is different in prokaryotic and eukaryotic organisms $[2,3]$.

However, the molecular details of the transcription processes in the pro- and eukaryotic organisms are still at its infancy. In this work, we tried to analyze the different aspects of the transcription factors from pro- and eukaryotic organisms. For the comparison purposes, we used the amino acid sequences of the DNA binding proteins (DBPs) and transcription factors (TFs) from UniProt [4].

We compared the TFs using their sequence information only as sequence is more abundant than structure [5]. The main ISSN 0973-2063 (online) 0973-8894 (print) motivation of carrying out the work is to discriminate between the different classes of microorganisms. We, for the first time, put forward some plausible discriminatory features between the TFs from the different branches of organisms. Interestingly, the TFs from the pro- and eukaryotic organisms can be distinctly identified using the amino acid frequency analyzes in the TFs. We also analyzed the statistical efficacies of the features used in the study to discriminate between the different classes of microorganisms using machine-learning techniques. The ideas regarding these features may further be utilized to come up with a prediction system to discriminate between the different branches of organisms.

\section{Methodology:}

Data collection:

We downloaded the sequences of DNA binding proteins (DBPs) from UniProt [4]. We collected the amino acid sequences of the DNA binding proteins from 1012 prokaryotic organisms and 1425 eukaryotes. We divided our dataset into two groups, the largest group containing the whole DBP data, and a small subgroup

BIOMEDICAL

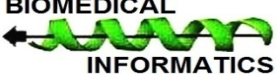


containing the transcription factor (TF) sequences, which were also present in the DNA binding protein dataset. The data collection process was carried out using an in-house tool written in Python (Figure 1).

\section{Redundancy check to the dataset:}

The raw dataset may be biased because of having multiple copies of a single sequence. We, therefore, performed a redundancy check, by means of distance matrix calculation. The distance matrix was generated by Hamming distance algorithm [6, 7]. After this redundancy check, we were able to eliminate the redundancy in the dataset and prepared a clean dataset. The clean dataset contained 270 DBP sequences from prokaryotes and 347 DBP sequences from eukaryotes; among them, there were 92 sequences of TF from prokaryotes and 182 sequences of TF from eukaryotes. So the DBP dataset contained 270 prokaryotic and 347 eukaryotic sequences. As the eukaryotic DBP sequences were present in higher number than the prokaryotic DBP sequences, we had split the eukaryotic DBP sequences into two sets. Eukaryotic DBP set 1 contained sequences starting from 1 to 270 and eukaryotic DBP and set 2 contained sequences starting from 78 to 347 so that there were equal numbers of amino acid sequences in the datasets. For the same reason, the eukaryotic TF dataset was split into two sets. TF set 1 contained sequences starting from 1 to 92 and TF set 2 contained sequences starting from 91 to 182. Thus all the datasets were balanced. The distribution of the dataset is shown in Table $\mathbf{1}$.

Table1: The distribution of the dataset.

\begin{tabular}{lclc}
\hline $\begin{array}{l}\text { DNA Binding Protein (DBP) } \\
\text { dataset }\end{array}$ & $\begin{array}{l}\text { Transcription Factor (TF) } \\
\text { Dataset }\end{array}$ \\
\hline $\begin{array}{l}\text { Prokaryote } \\
1-270\end{array}$ & Eukaryote Set-1 & Prokaryote & Eukaryote Set-1 \\
& $1-270$ & $1-92$ & $1-92$ \\
& Eukaryote Set-2 & & Eukaryote Set-2 \\
$78-347$ & & $91-182$ \\
\hline
\end{tabular}

The list of UniProt IDs used in these datasets was present in Table S1 (see Supplementary data).

\section{Frequency Calculation:}

After the preparation of these clean datasets, we performed amino acids and amino acids group frequency calculations. We categorized the amino acid groups into Hydrophobic (HB), Hydrophilic (HI), Charged (CR), Basic (BS) and Acidic (AC) [8]. This frequency calculation was done to normalize the dataset. The entire frequency calculation was done using an in-house python script. We had calculated the frequency of amino acids and amino acid groups separately for the two datasets DBP and $\mathrm{TF}$, and separately for eukaryotic set1 and eukaryotic set 2 .

\section{Machine learning using WEKA:}

We used the overall amino acid frequencies and amino acids group frequencies of the prokaryotic and eukaryotic organisms as features to distinguish between prokaryotic and eukaryotic organisms using the tool WEKA [9]. WEKA is a tool, containing a collection of machine learning algorithms, is commonly used in data mining problems in bioinformatics. We have used the
Support vector machine (SVM) algorithm and the SMO classifier [10] with 10 fold cross-validation. The 10 fold cross validation is a kind of default test option of WEKA. It randomly splits the dataset into training and testing datasets and runs the test. It does this operation 10 times with random splitting of the input data into training and testing datasets. We prepared the input dataset for WEKA using data distribution as described in table $\mathbf{1}$.

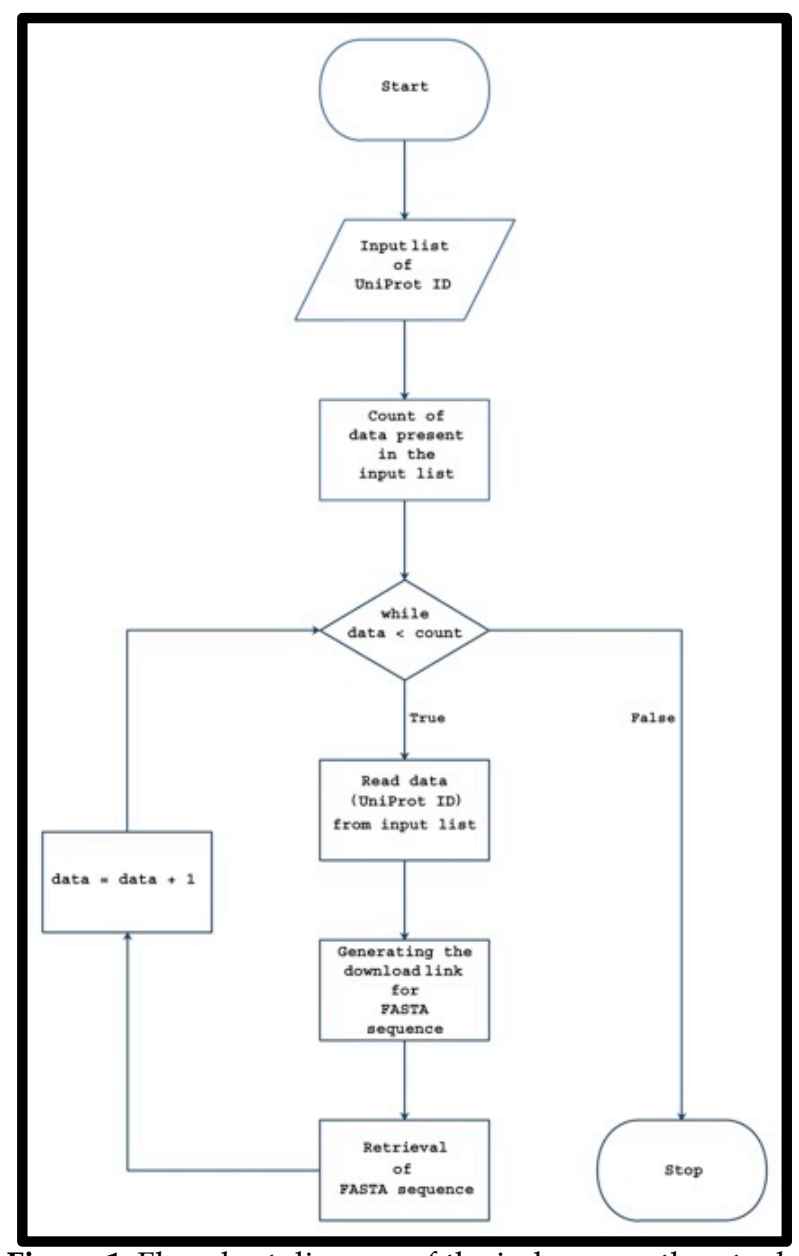

Figure 1: Flowchart diagram of the in-house python tool.

\section{Results:}

Amino acids and amino acid group frequency

A distinguishable difference was found in the frequency patterns between eukaryotic and prokaryotic amino acid sequences in the DNA binding proteins. This distinguishable difference pattern in amino acid and amino acid group frequency can be used to discriminate them. The bar graph (Figure 2) and boxplot (Figure 3 and Figure 4) were used to decipher the patterns of the differences.

\section{Machine learning results:}

We found that amino acids and amino acid group frequency can be used as features to train a SMO classifier in WEKA to distinguish prokaryotic and eukaryotic DNA binding proteins on 
the basis of their amino acid and amino acid group frequency as given in Table 2.

Table 2: Results obtained from WEKA analysis.

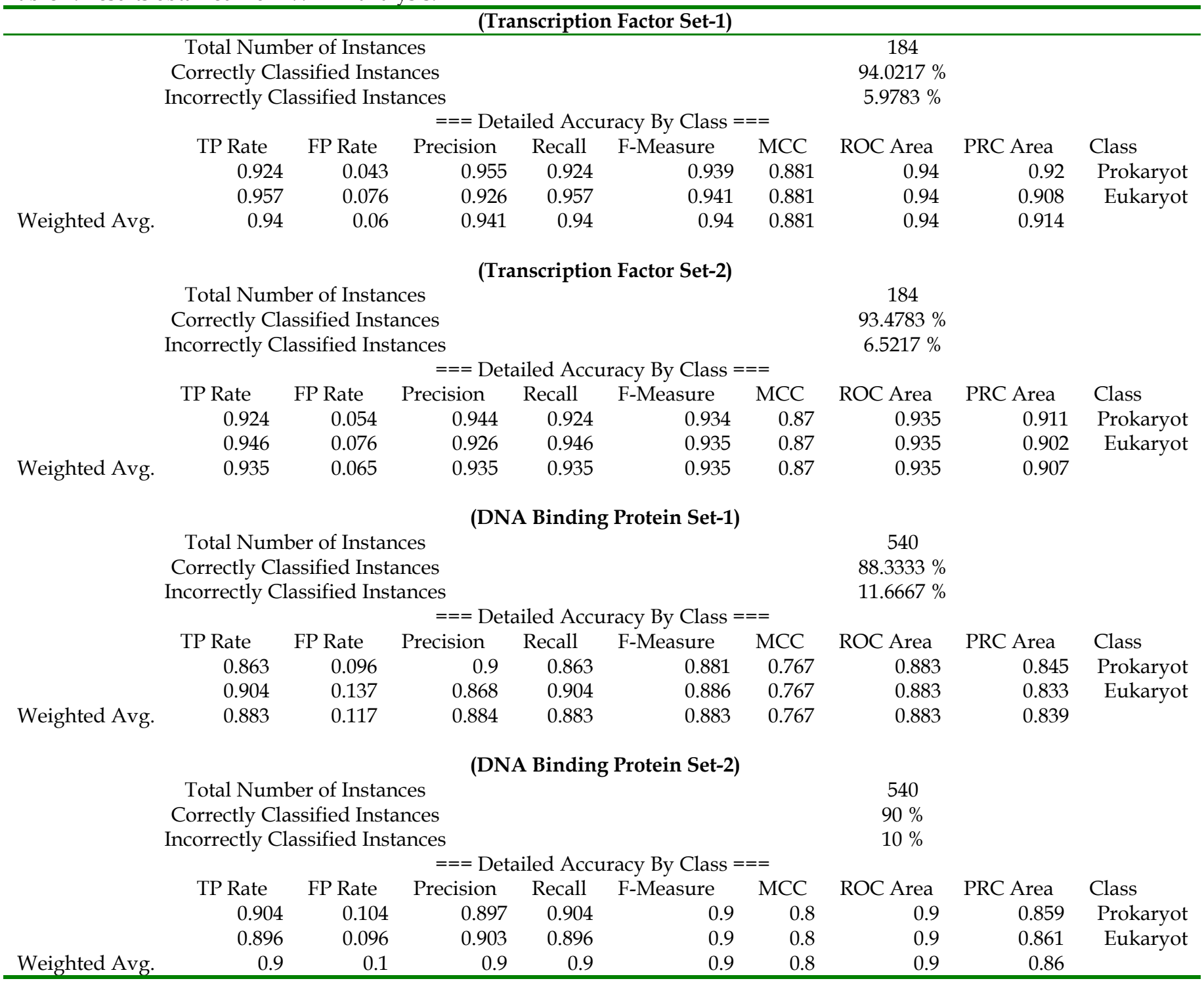

\section{Discussion:}

Data show that the sequence-based features of the DBPs and TFs could very well be used to distinguish between these classes of organisms. In all our analyses, we obtained an overall accuracy greater than $85 \%$ and an AUC value of 0.9 . However, we had to use a comparatively small dataset due to paucity of data in the databases. None-the-less, this is the up to date data available till the date mentioned in the manuscript. Available predictors combine both the sequence and structural information for the discrimination purposes. Our predictor uses only sequence information and therefore may be considered a more general one as sequence information is more abundant than structural information. For extraction of the features, we used an in-house script written in python.

\section{Acknowledgment:}

The authors acknowledge University of Kalyani, Kalyani (W.B.) India and DBT funded Bioinformatics Infrastructure Facility (BIF) for providing infrastructure facilities. NC would like thank the UGC, Govt. of India for financial support. The infrastructural supports from DBT, India (San No. 102/IFD/SAN/1822/20152016 dated August 06 2015) and ICMR (BIC/12(02)/2014), Govt. of India are duly acknowledged. 


\section{BIOINFORMATION \\ Discovery at the interface of physical and biological sciences}

Open access

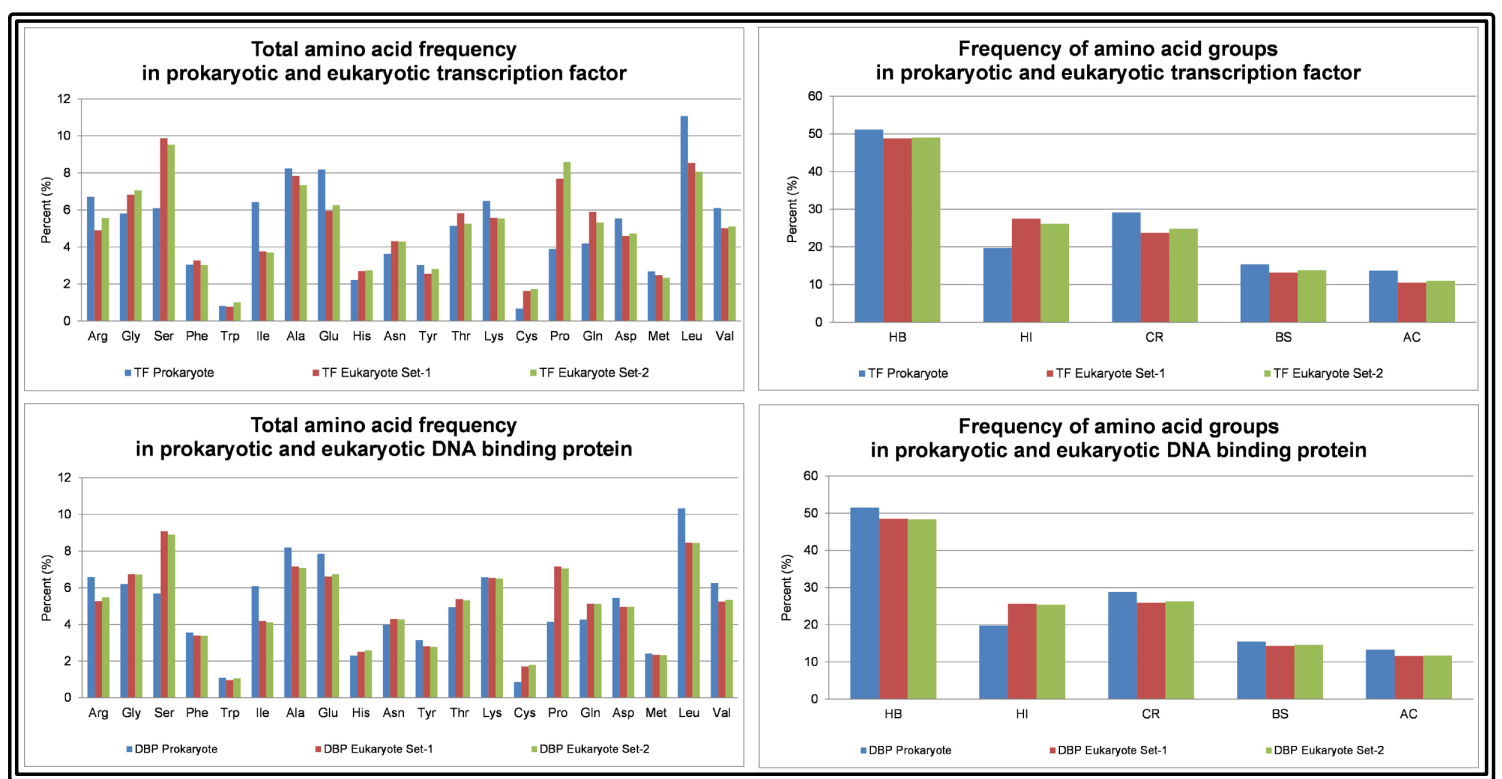

Figure 2: The bar-graph representation of amino acids and amino acid group frequency in prokaryotes and eukaryotes (Blue: Prokaryote; Red: Eukaryote Set-1; Green: Eukaryote Set-2).

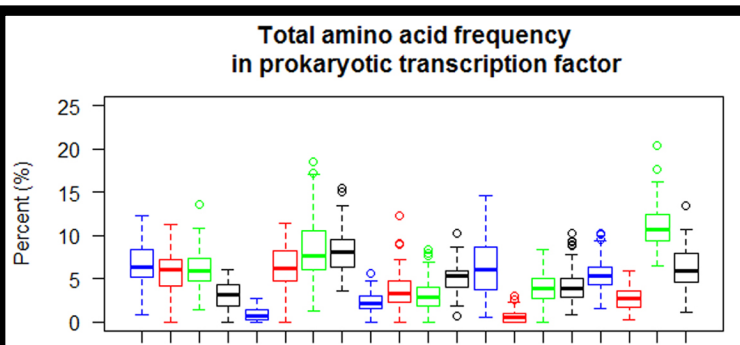

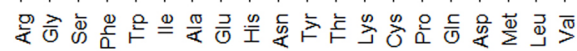

Total amino acid frequency in eukaryotic transcription factor (Set-1)

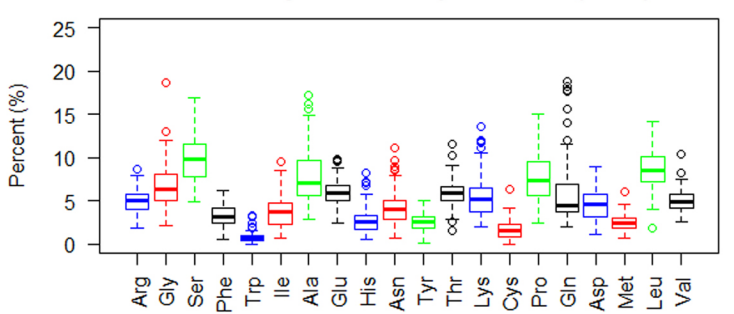

Total amino acid frequency in eukaryotic transcription factor (Set-2)

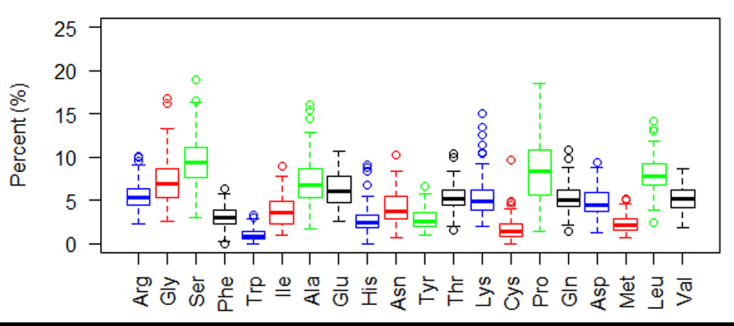

Frequency of amino acid groups in prokaryotic transcription factor

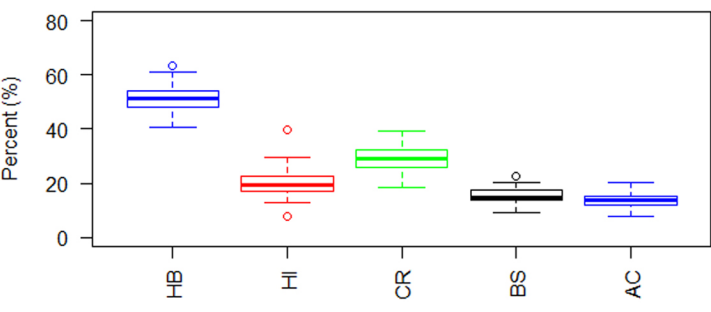

Frequency of amino acid groups in eukaryotic transcription factor (Set-1)

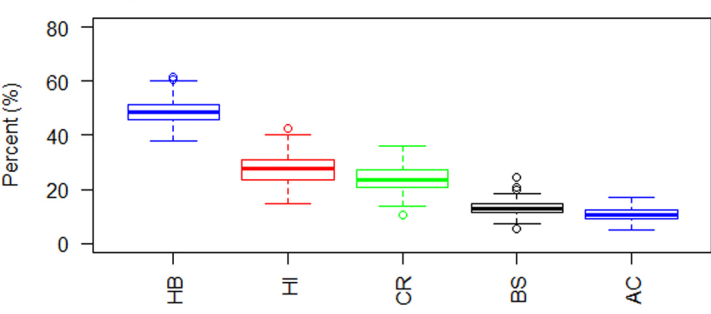

Frequency of amino acid

groups in eukaryotic transcription factor (Set-2)

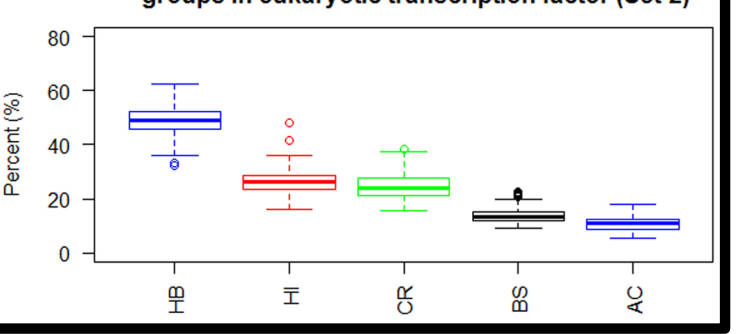

Figure 3: Amino acids and amino acid group frequency from TF dataset.

ISSN 0973-2063 (online) 0973-8894 (print)

Bioinformation 14(6): 315-326 (2018) 


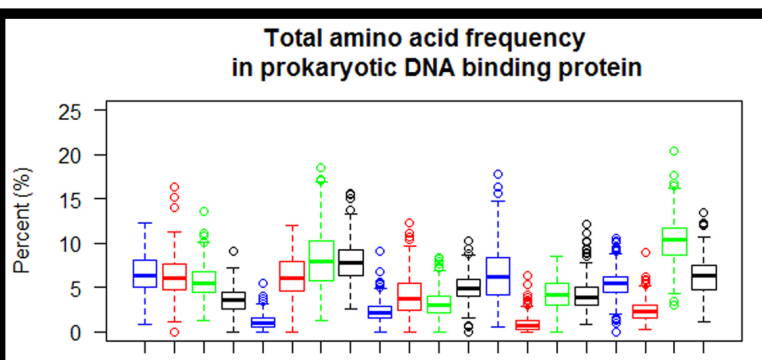

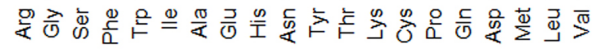

Total amino acid frequency in eukaryotic DNA binding protein (Set-1)

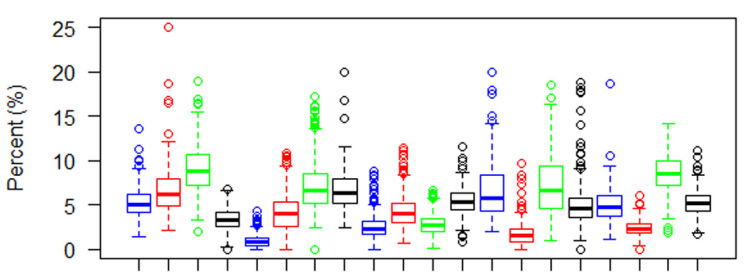

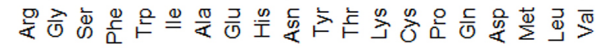

Total amino acid frequency in eukaryotic DNA binding protein (Set-2)

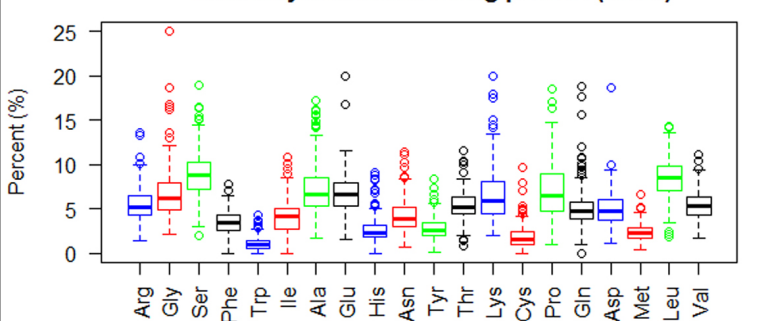

Frequency of amino acid

groups in prokaryotic DNA binding protein

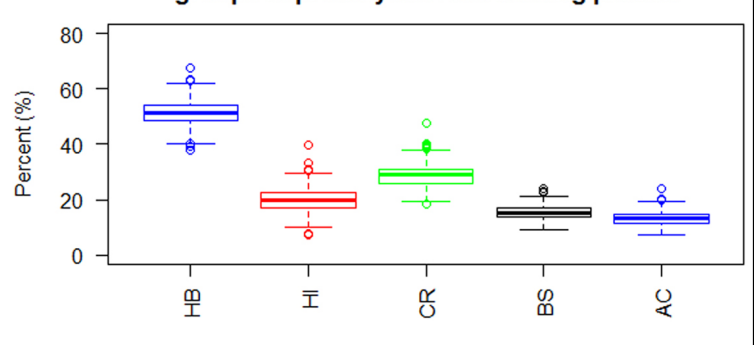

Frequency of amino acid groups in eukaryotic DNA binding protein (Set-1)

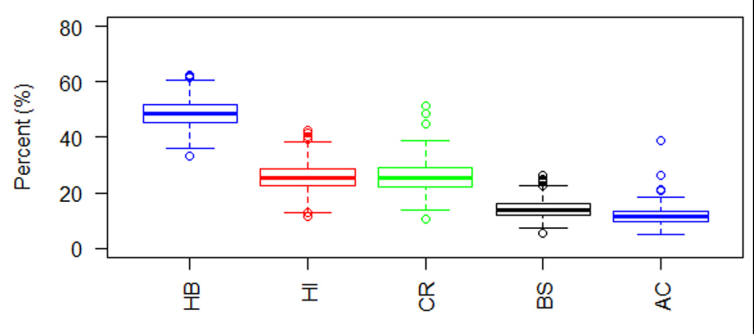

Frequency of amino acid groups in eukaryotic DNA binding protein (Set-2)

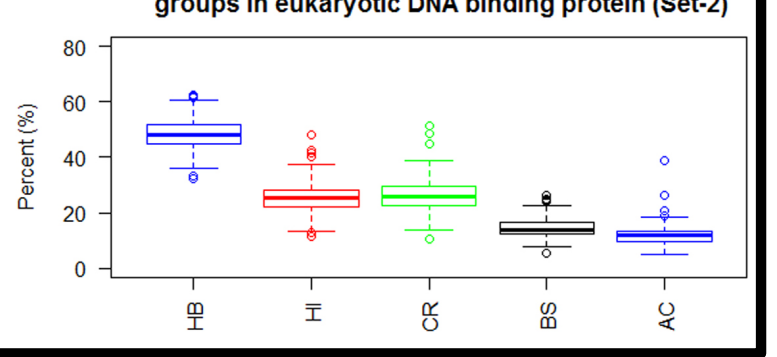

Figure 4: Amino acids and amino acid group frequency from DBP dataset.

\section{References:}

[1] Latchman DS. International Journal of Biochemistry and Cell Biology. 1997, 29:1305. [PMID: 9570129]

[2] Spitz \& Furlong, Nat. Rev. Genet. 2012, 13:613. [PMID: 22868264]

[3] Bagchi A. Gene. 2016, 586:274 [PMID: 27083770]

[4] UniProt Consortium, Nucleic Acids Res. 2013, 41:D43. [PMID: 23161681]

[5] Al-Shahib A et al. BMC Genomics. 2007, 8:78 [PMID: 17374164]
[6] Hamming RW. Bell Syst. Tech. J. 1950, 29:147.

[7] Blackburne BP and Whelan S. Bioinformatics. 2012, 28:495. [PMID: 22199391]

[8] Nelson DL \& Cox MM. Lehninger Principles of Biochemistry. 2005, 4.

[9] Frank E et al. "Weka," in Data Mining and Knowledge Discovery Handbook, 2005, 1305.

[10] Frank E et al. Bioinformatics. 2004, 20:2479 [PMID: 15073010]

Edited by $P$ Kangueane

Citation: Chowdhury \& Bagchi. Bioinformation 14(6): 315-326 (2018)

License statement: This is an Open Access article which permits unrestricted use, distribution, and reproduction in any medium, provided the original work is properly credited. This is distributed under the terms of the Creative Commons Attribution License

ISSN 0973-2063 (online) 0973-8894 (print)

Bioinformation 14(6): 315-326 (2018)
BIOMEDICAL

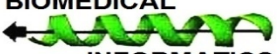

INFORMATICS 


\section{Supplementary Data:}

Table S1: List of UniProt id of the FASTA files used as dataset

\begin{tabular}{|c|c|c|c|}
\hline $\begin{array}{l}\text { Prokaryotic } \\
\text { TF }\end{array}$ & $\begin{array}{l}\text { Eukaryotic } \\
\text { TF }\end{array}$ & $\begin{array}{l}\text { Prokaryotic } \\
\text { DBP }\end{array}$ & $\begin{array}{l}\text { Eukaryotic } \\
\text { DBP }\end{array}$ \\
\hline A0A0H2VJZ8 & A0AVK6 & A0A072Z681 & A0AVK6 \\
\hline A0QZ11 & A2D9X4 & A0A0H2VJZ8 & A0JP82 \\
\hline A0R6I8 & G0SB31 & A0A0H2XIU6 & A2D9X4 \\
\hline A6T8N1 & G4NEJ8 & A0QZ11 & A5J036 \\
\hline B2SU53 & L7I1M8 & A0R6I8 & A6ZL36 \\
\hline B8FW11 & O00327 & A3DJ38 & B4F6I0 \\
\hline C3W947 & O00482 & A3FMN7 & C0JWR6 \\
\hline D5KM69 & O15350 & A5TY69 & C7SWF3 \\
\hline G3XCY4 & O15409 & A6T8N1 & D2W6T1 \\
\hline O34777 & O43435 & B2MU09 & D9IWL3 \\
\hline O34817 & O43524 & B2SU53 & D9J034 \\
\hline O66551 & O54790 & B8FW11 & E0YCK3 \\
\hline O66858 & O94916 & C1D7P6 & F7WD42 \\
\hline O68014 & O95238 & C3W947 & G0SB31 \\
\hline O69245 & P01100 & D4EMQ0 & G4NEJ8 \\
\hline P03023 & P01106 & D5KM69 & L7I1M8 \\
\hline P03052 & P02340 & D5MNX7 & M1GSK9 \\
\hline P06533 & P02833 & D9N168 & O00327 \\
\hline P06534 & P02836 & E1C9K5 & O00482 \\
\hline P07674 & P03001 & G3XCY4 & O13988 \\
\hline P0A0I7 & P03069 & O25100 & O14770 \\
\hline P0A0N4 & P03372 & O25386 & O14862 \\
\hline P0A247 & P04150 & O25758 & O15350 \\
\hline Р0A4T9 & P04386 & O25841 & O15409 \\
\hline P0A6X7 & P04637 & O34777 & O15527 \\
\hline P0A881 & P05412 & O34817 & O43435 \\
\hline P0A8U6 & P05554 & O52512 & O43524 \\
\hline P0A8V6 & P05725 & O66551 & O54790 \\
\hline POACIO & P06536 & O66659 & O74859 \\
\hline P0ACJ8 & P06601 & O66858 & O75362 \\
\hline P0ACP7 & P06602 & O68014 & O75531 \\
\hline P0ACS2 & P07270 & O68557 & O80358 \\
\hline P0ACT4 & P07272 & O68847 & O82175 \\
\hline P0AF28 & P08046 & O69245 & O94468 \\
\hline P0AFJ5 & P08151 & O83028 & O94916 \\
\hline P0AG30 & P08638 & O87365 & O95238 \\
\hline P0AGK8 & P09077 & O87963 & O95243 \\
\hline P0C1U6 & P09631 & P00582 & O95551 \\
\hline P0DJL7 & P09956 & P00642 & P00639 \\
\hline P10026 & P0CS82 & P00648 & P00734 \\
\hline P17893 & P0CY08 & P02958 & P01100 \\
\hline P21866 & P0CY10 & P03004 & P01106 \\
\hline P22262 & P10037 & P03013 & P01127 \\
\hline P23873 & P10085 & P03018 & P01837 \\
\hline P23874 & P10276 & P03023 & P02263 \\
\hline P25144 & P11473 & P03052 & P02340 \\
\hline P27709 & P11831 & P03067 & P02833 \\
\hline P33905 & P11938 & P03856 & P02836 \\
\hline P39075 & P13297 & P04390 & P03001 \\
\hline P40676 & P13393 & P04395 & P03069 \\
\hline
\end{tabular}

ISSN 0973-2063 (online) 0973-8894 (print) 


\section{BIOINFORMATION}

Discovery at the interface of physical and biological sciences

\begin{tabular}{|c|c|c|c|}
\hline P44558 & P14859 & P04995 & P03372 \\
\hline P46828 & P14921 & P05050 & P03870 \\
\hline P68261 & P15036 & P05102 & P03880 \\
\hline P71039 & P15207 & P05327 & P03882 \\
\hline P96711 & P15806 & P05523 & P04150 \\
\hline P9WGZ1 & P16236 & P06134 & P04275 \\
\hline P9WJB7 & P17676 & P06533 & P04386 \\
\hline P9WME9 & P17679 & P06534 & P04637 \\
\hline P9WMF8 & P17789 & P06612 & P05231 \\
\hline P9WMH1 & P18113 & P07013 & P05412 \\
\hline P9WMH3 & P19419 & P07674 & P05554 \\
\hline P9WPY9 & P19544 & P08394 & P05725 \\
\hline Q0P6M2 & P19793 & P09184 & P06401 \\
\hline Q1D4I5 & P19838 & P09546 & P06536 \\
\hline Q2ACK9 & P20153 & P09883 & P06601 \\
\hline Q2FZ56 & P20226 & P09980 & P06602 \\
\hline Q32WH4 & P20263 & P0A0I7 & P06766 \\
\hline Q3ZD72 & P20393 & P0A0N4 & P06786 \\
\hline Q45782 & P20823 & Р0A247 & P07199 \\
\hline Q46731 & P21952 & P0A459 & P07270 \\
\hline Q46864 & P22121 & Р0А4T9 & P07272 \\
\hline Q57468 & P22415 & P0A6C1 & P07276 \\
\hline Q5F882 & P22670 & P0A6R3 & P08046 \\
\hline Q5Y812 & P22829 & P0A6Z6 & P08151 \\
\hline Q746J7 & P23511 & P0A7C2 & P08638 \\
\hline Q7AKF2 & P23760 & P0A7G6 & P09077 \\
\hline Q7X0D9 & P23772 & P0A809 & P09631 \\
\hline Q83TD2 & P24781 & P0A881 & P09651 \\
\hline Q8AAV8 & P25490 & P0A8J2 & P09838 \\
\hline Q8E565 & P25502 & P0A8U6 & P09874 \\
\hline Q8GGH0 & P25799 & P0A8V6 & P09884 \\
\hline Q8NMG3 & P27577 & P0A988 & P09956 \\
\hline Q8YAF1 & P28147 & P0A9H1 & P0CS82 \\
\hline Q933Z0 & P28324 & P0ABS5 & P0CY08 \\
\hline Q9CHR1 & P28347 & P0AC51 & P0CY10 \\
\hline Q9EZJ8 & P29617 & P0ACI0 & P10037 \\
\hline Q9HUS3 & P31266 & P0ACJ8 & P10085 \\
\hline Q99I1S1 & P34707 & P0ACP7 & P10276 \\
\hline Q9KQU8 & P35680 & P0ACS2 & P11308 \\
\hline Q9KWU8 & P35869 & P0ACT4 & P11387 \\
\hline Q9S166 & P36956 & P0ADI2 & P11473 \\
\hline \multirow[t]{16}{*}{ Q9Z9H6 } & P38144 & P0AEE8 & P11831 \\
\hline & P38830 & P0AEK0 & P11938 \\
\hline & P38867 & P0AF28 & P12689 \\
\hline & P41235 & P0AFJ5 & P12956 \\
\hline & P42226 & P0AFY8 & P13051 \\
\hline & P42227 & P0AG30 & P13297 \\
\hline & P42582 & P0AG74 & P13393 \\
\hline & P43680 & P0AGE0 & P13864 \\
\hline & P46531 & P0AGK8 & P14585 \\
\hline & P47902 & P0C1U6 & P14653 \\
\hline & P48436 & P0CI76 & P14736 \\
\hline & P49711 & P0DJL7 & P14859 \\
\hline & P51608 & P0DJO8 & P14921 \\
\hline & P52952 & P11405 & P15036 \\
\hline & P53539 & P13920 & P15207 \\
\hline & P53762 & P13925 & P15424 \\
\hline
\end{tabular}

ISSN 0973-2063 (online) 0973-8894 (print) 


\section{BIOINFORMATION}

Discovery at the interf ace of physical and biological sciences

\begin{tabular}{|c|c|c|}
\hline P53999 & P14294 & P15436 \\
\hline P54841 & P14385 & P15806 \\
\hline P55318 & P14565 & P15919 \\
\hline P56178 & P14633 & P16236 \\
\hline P61244 & P14870 & P16455 \\
\hline P70118 & P15005 & P17255 \\
\hline P70340 & P15042 & P17542 \\
\hline P70348 & P16525 & P17676 \\
\hline P70512 & P17743 & P17679 \\
\hline P83949 & P17888 & P17789 \\
\hline P84022 & P17893 & P18113 \\
\hline P87249 & P19821 & P18858 \\
\hline P97360 & P20384 & P19419 \\
\hline P97471 & P20589 & P19544 \\
\hline P98177 & P21189 & P19793 \\
\hline Q00059 & P21338 & P19838 \\
\hline Q00403 & P21866 & P20153 \\
\hline Q00422 & P22262 & P20226 \\
\hline Q00613 & P23478 & P20263 \\
\hline Q00653 & P23657 & P20393 \\
\hline Q00958 & P23873 & P20823 \\
\hline Q01147 & P23874 & P21951 \\
\hline Q01167 & P23909 & P21952 \\
\hline Q01543 & P23940 & P22121 \\
\hline Q01663 & P25144 & P22415 \\
\hline Q001826 & P27709 & P22670 \\
\hline Q02078 & P28630 & P22829 \\
\hline Q02080 & P30014 & P23511 \\
\hline Q02548 & P31032 & P23760 \\
\hline Q03347 & P33788 & P23772 \\
\hline Q04206 & P33905 & P23906 \\
\hline Q04207 & P37954 & P24781 \\
\hline Q04863 & P39075 & P25490 \\
\hline Q05195 & P40676 & P25502 \\
\hline Q06330 & P41016 & P25799 \\
\hline Q06831 & P42371 & P26358 \\
\hline Q08050 & P43642 & P26367 \\
\hline Q089957 & P43870 & P26368 \\
\hline Q12778 & P44558 & P27577 \\
\hline Q13148 & P44688 & P27694 \\
\hline Q13469 & P46828 & P27695 \\
\hline Q14653 & P50187 & P28147 \\
\hline Q14863 & P50465 & P28324 \\
\hline Q14919 & P52026 & P28347 \\
\hline Q15561 & P56255 & P28519 \\
\hline Q16254 & P56981 & P29372 \\
\hline Q16666 & P62558 & P29549 \\
\hline Q17034 & P68261 & P29617 \\
\hline Q3UPW2 & P70985 & P31266 \\
\hline Q58HP3 & P71039 & P31483 \\
\hline Q5AP80 & P72525 & P31941 \\
\hline Q60793 & P76116 & P32657 \\
\hline Q61473 & P83847 & P32761 \\
\hline Q64249 & P84131 & P34257 \\
\hline Q6MZP7 & P96711 & P34707 \\
\hline Q6NT76 & P96856 & P35680 \\
\hline Q8C6P8 & P9WGZ1 & P35869 \\
\hline
\end{tabular}

ISSN 0973-2063 (online) 0973-8894 (print) 


\section{BIOINFORMATION}

Discovery at the interface of physical and biological sciences

\begin{tabular}{|c|c|c|}
\hline Q8GZB6 & P9WII3 & P36956 \\
\hline Q8IKH2 & P9WJB7 & P38144 \\
\hline Q8L7G0 & P9WME9 & P38830 \\
\hline Q8MXE7 & P9WMF8 & P38867 \\
\hline Q8NHW3 & P9WMH1 & P39748 \\
\hline Q94702 & P9WMH3 & P41235 \\
\hline Q94IF5 & P9WNV3 & P42224 \\
\hline Q95VR4 & P9WPY9 & P42226 \\
\hline Q969G2 & Q031W6 & P42227 \\
\hline Q99551 & Q06B24 & P42582 \\
\hline Q99626 & Q0P6M2 & P43246 \\
\hline Q9C932 & Q1D4I5 & P43680 \\
\hline Q9H3D4 & Q2ACK9 & P46531 \\
\hline Q9NQV7 & Q2FZ56 & P47902 \\
\hline Q9NUX5 & Q2I6W2 & P48436 \\
\hline Q9UHX1 & Q32WH4 & P49711 \\
\hline Q9UMN6 & Q3ZD72 & P49916 \\
\hline \multirow[t]{40}{*}{ Q9Y5R6 } & Q45458 & P50534 \\
\hline & Q45488 & P50549 \\
\hline & Q45782 & P51608 \\
\hline & Q46731 & P52952 \\
\hline & Q46864 & P53539 \\
\hline & Q46896 & P53762 \\
\hline & Q46944 & P53999 \\
\hline & Q47112 & P54098 \\
\hline & Q47152 & P54132 \\
\hline & Q47155 & P54274 \\
\hline & Q47673 & P54841 \\
\hline & Q47PJ0 & P55265 \\
\hline & Q4UNB2 & P55318 \\
\hline & Q53632 & P56178 \\
\hline & Q56215 & P60896 \\
\hline & Q57253 & P61244 \\
\hline & Q57267 & P61823 \\
\hline & Q57468 & P61978 \\
\hline & Q5F882 & P62805 \\
\hline & Q5F9M9 & P63159 \\
\hline & Q5I6E6 & P70118 \\
\hline & Q5KWC1 & P70340 \\
\hline & Q5L0J3 & P70348 \\
\hline & Q5SJ64 & P70512 \\
\hline & Q5SJ65 & P83949 \\
\hline & Q5SJC4 & P84022 \\
\hline & Q5Y812 & P87249 \\
\hline & Q72I39 & P97360 \\
\hline & Q746J7 & P97471 \\
\hline & Q746M7 & P98177 \\
\hline & Q7AKF2 & Q00059 \\
\hline & Q7CWV1 & Q00403 \\
\hline & Q7DD47 & Q00422 \\
\hline & Q7MHK3 & Q00613 \\
\hline & Q7X0D9 & Q00653 \\
\hline & Q816E8 & Q00958 \\
\hline & Q83TD2 & Q01147 \\
\hline & Q84AF2 & Q01167 \\
\hline & Q8AAV8 & Q01543 \\
\hline & Q8DPM2 & Q01663 \\
\hline
\end{tabular}

ISSN 0973-2063 (online) 0973-8894 (print) 
BIOINFORMATION

Discovery at the interface of physical and biological sciences
Open access

\begin{tabular}{ll} 
Q8E565 & Q01826 \\
Q8EFJ3 & Q02078 \\
Q8EIX3 & Q02080 \\
Q8EVR5 & Q02486 \\
Q8GGH0 & Q02548 \\
Q8KNP2 & Q02880 \\
Q8NMG3 & Q03164 \\
Q8R5T9 & Q03347 \\
Q8RNV5 & Q04049 \\
Q8RNV8 & Q04206 \\
Q8RT53 & Q04207 \\
Q8YAF1 & Q04863 \\
Q8Z2A5 & Q05195 \\
Q8ZG78 & Q05783 \\
Q928V6 & Q06330 \\
Q933Z0 & Q06453 \\
Q93PU6 & Q06831 \\
Q97FM4 & Q07230 \\
Q99U17 & Q08050 \\
Q9AC34 & Q08874 \\
Q9AFI5 & Q08957 \\
Q9AMH9 & Q12778 \\
Q9CHR1 & Q13469 \\
Q9EZJ8 & Q13569 \\
Q9F6L0 & Q14191 \\
Q9HUS3 & Q14653 \\
Q9I0M3 & Q14863 \\
Q9IS1 & Q14919 \\
Q9I2N0 & Q15109 \\
Q9KEI9 & Q15365 \\
Q9KJ88 & Q15366 \\
Q9KQU8 & Q15554 \\
Q9KVD2 & Q15561 \\
Q9KWU8 & Q16254 \\
Q9KXR9 & Q16531 \\
Q9RPJ3 & Q16666 \\
Q9RT63 & Q17034 \\
Q9RWH8 & Q25442 \\
Q9RY80 & Q3UPW2 \\
Q9S166 & Q4PRK9 \\
Q9WY48 & Q4VWW5 \\
Q9WYV0 & Q58HP3 \\
Q9X2H9 & Q5AP80 \\
Q9X4C9 & Q5EAW4 \\
Q9XDH5 & Q5NE14 \\
Q9Z3B4 & Q5XJA0 \\
Q9Z9H6 & Q60793 \\
Q9ZL26 & Q61473 \\
V6F4Q0 & Q64249 \\
& Q68E01 \\
& \\
& Q6PM73 \\
\hline
\end{tabular}

ISSN 0973-2063 (online) 0973-8894 (print) 
Q7JQ07

Q7M3K2

Q7T2M9

Q7TS98

Q7Z2E3

Q7Z5Q5

Q84KJ5

Q84ZU4

Q86T24

Q8C6L5

Q8C6P8

Q8GZB6

Q8IKH2

Q8L7G0

Q8MXE7

Q8N5Y2

Q8NHW3

Q8SXK5

Q8SYK5

Q8VDF2

Q91VJ1

Q91XB0

Q921F2

Q92383

Q94702

Q94IF5

Q95VR4

Q969G2

Q96LI5

Q96LW4

Q96PU4

Q96T88

Q99551

Q99626

Q9C932

Q9DFY5

Q9GPZ9

Q9H171

Q9H3D4

Q9H9S0

Q9JIW4

Q9JJX7

Q9JLV6

Q9NP87

Q9NQV7

Q9NUW8

Q9NUX5

Q9P016

Q9P0U4

Q9QY24

Q9R002

Q9R1E6

Q9UBT6

Q9UBZ9

Q9UGP5

Q9UH17

Q9UHX1 
Q9UMN6

Q9UNA4

Q9UQ84

Q9UTN9

Q9VD99

Q9VR17

Q9Y253

Q9Y261

Q9Y2M0

Q9Y5R6

Q9YGN6

Q9Z2D7 